\title{
Microbiological, clinical and molecular findings of non-typhoidal Salmonella bloodstream infections associated with malaria, Oriental Province, Democratic Republic of the Congo
}

Dadi Falay ${ }^{1+}$, Laura Maria Francisca Kuijpers ${ }^{2,3^{*}}$, Marie-France Phoba ${ }^{4}$, Hilde De Boeck ${ }^{2}$, Octavie Lunguya ${ }^{4}$, Emmanuel Vakaniaki ${ }^{5}$, Sophie Bertrand ${ }^{6}$, Wesley Mattheus ${ }^{6}$, Pieter-Jan Ceyssens ${ }^{6}$, Raymond Vanhoof ${ }^{6}$, Hugo Devlieger ${ }^{7}$, Chris Van Geet ${ }^{7}$, Erik Verheyen ${ }^{8,9}$, Dauly Ngbonda ${ }^{1}$ and Jan Jacobs ${ }^{2,3}$

\begin{abstract}
Background: In sub-Saharan Africa, non-typhoidal Salmonella (NTS) can cause bloodstream infections, referred to as invasive non-typhoidal Salmonella disease (iNTS disease); it can occur in outbreaks and is often preceded by malaria. Data from Central Africa is limited.

Methods: Clinical, microbiological and molecular findings of NTS recovered in a blood culture surveillance project (2009-2014) were analyzed.

Results: In March-July 2012 there was an epidemic increase in malaria infections in the Oriental Province of the Democratic Republic of the Congo (DRC). In one referral hospital, overall hospital admissions in June 2012 were 2.6 times higher as compared to the same period in the years before and after (336 versus an average of 128 respectively); numbers of malaria cases and blood transfusions were nearly three- and five-fold higher respectively (317 versus 112 and 250 versus 55). Case fatality rates (in-hospital deaths versus all admissions) peaked at $14.6 \%$. Salmonella Typhimurium and Salmonella Enteritidis together accounted for $88.9 \%$ of pathogens isolated from blood cultures collected during an outreach visit to the affected districts in June 2012. Children infected with Salmonella Enteritidis (33 patient files available) tended to be co-infected with Plasmodium falciparum more often than children infected with Salmonella Typhimurium (40 patients files available) (81.8\% versus $62.5 \%$ ). Through the microbiological surveillance project (May 2009-May 2014) 113 unique NTS isolates were collected (28.5 \% (113/396) of pathogens); most (95.3\%) were recovered from children $<15$ years. Salmonella Typhimurium $(n=54)$ and Salmonella Enteritidis $(n=56)$ accounted for $47.8 \%$ and of $49.6 \%$ NTS isolates respectively. Multilocus variable-number tandem-repeat analysis (MLVA) revealed more heterogeneity for Salmonella Typhimurium than for Salmonella Enteritidis. Most (82/96, $85.4 \%$ ) NTS isolates that were available for antibiotic susceptibility testing were multidrug resistant. All isolates were susceptible to ceftriaxone and azithromycin.

(Continued on next page)
\end{abstract}

\footnotetext{
* Correspondence: Ikuijpers@itg.be

${ }^{\dagger}$ Equal contributors

${ }^{2}$ Department of Clinical Sciences, Institute of Tropical Medicine, Antwerp,

Belgium

${ }^{3}$ Department of Microbiology and Immunology, KU Leuven, Leuven, Belgium

Full list of author information is available at the end of the article
} 
(Continued from previous page)

Conclusion: During the peak of an epidemic increase in malaria in the DRC in 2012, a high proportion of multidrug resistant Salmonella Typhimurium and Salmonella Enteritidis were isolated from blood cultures. Overall, the two serovars showed subtle differences in clinical presentation and genetic diversity.

Keywords: Bloodstream infections, Salmonella, Democratic Republic of the Congo, Antibiotic, Molecular typing, Symptoms, Children, Malaria

\section{Background}

In industrialized countries, non-typhoidal Salmonella (NTS, i.e., Salmonella serovars other than Salmonella enterica serovar Typhi and Salmonella enterica serovar Paratyphi) are associated with self-limiting diarrhea. In sub-Saharan Africa however, they often cause bloodstream infections, which is referred to as invasive nontyphoidal Salmonella disease (iNTS disease). They are among the pathogens most frequently isolated from blood cultures [1-4]. In fact, iNTS disease poses an important public health problem with an estimated population-based annual incidence of 175-388 cases/ 100,000 in children aged 3-5 years of age [2]. Due to the lack of microbiological facilities in many African countries, the true burden of iNTS disease is not well known and the condition remains undiagnosed and hence invisible and neglected.

iNTS disease occurs mainly in children and immunecompromised individuals and a number of conditions make individuals more susceptible including malnutrition and Plasmodium falciparum malaria [5-9]. The two NTS serovars most prevalent in sub-Saharan Africa are Salmonella enterica serovar Typhimurium (hereafter referred to as Salmonella Typhimurium) and Salmonella enterica serovar Enteritidis (hereafter referred to as Salmonella Enteritidis).

Studies about NTS infections in children have been reported mainly from East, West and South Africa [1, 10-14]. Recently, epidemic increases of NTS infections were reported from the Democratic Republic of the Congo (DRC) in Central Africa; in Kisantu in 2011 (South West, Bas Congo Province) and in Bwamanda in 2012 (North West, Equatorial Province) [15, 16] (Fig. 1).

In April 2012, local health authorities in the district of Haut-Uélé (North East, Oriental Province) (Fig. 2) were alerted by significant increases in numbers of hospitals admissions, blood transfusions and case fatality rates among children. This observation was also made in the neighboring district of Bas-Uélé and was related to an increase of malaria infections as reported by different organizations at that time [17].

However, most children did not respond favorably to antimalarials but improved when antibiotics were administered. This raised suspicion of a concomitant increase in NTS infections as was previously witnessed in Bwamanda and Kisantu and resulted in the Ministry of Health recommending empirical treatment of intravenous quinine combined with antibiotics (ceftriaxone or ciprofloxacin) to all affected children. Blood culture sampling in the affected districts allowed assessment of a possible concomitant burden of invasive Salmonella infections. Due to logistic and administrative difficulties, sampling was only initiated from June 2012 onwards. Site visits also included reviewing of clinical charts of pediatric patients with invasive salmonellosis.

The present report aims to describe the clinical, microbiological, and molecular characteristics of the invasive Salmonella infections observed during this period of increased admissions and malaria infections in the Bas-Uélé and Haut-Uélé districts as well as of invasive Salmonella infections recorded in the entire Oriental Province in the period before and after 2012 (2009-2014).

\section{Methods \\ Study site}

The Oriental Province in the DRC covers an area of $503,239 \mathrm{~km}^{2}$ with 8,633,573 inhabitants and comprises four administrative districts: Tshopo (including Kisangani, province capital), Haut-Uélé, Bas-Uélé and Ituri. Each district is divided into health zones (HZs) with a General Referral Hospital. The four health zones mainly affected by an epidemic increase in malaria in 2012 were located in the districts Haut-Uélé (HZs of Wamba and Isiro) and Bas-Uélé (HZs of Pawa and Poko) (Fig. 2). Demographic data of these HZs are displayed in Table 1 . The majority of the population is living from agriculture, livestock, informal trade and small-scale mining [18]. There is a tropical rainforest climate with peaks of rainfall in April and October and a dryer season between November and February. Malaria is the leading cause of morbidity and mortality among children and P. falciparum is holoendemic with a perennial transmission [19].

During the year 2012, the Eastern DRC experienced increases in violence due to various armed groups leading to 466,000 displaced persons in the Oriental Province [20]. Apart from the reported increase in malaria infections, the province also faced outbreaks of cholera, measles and meningitis [21]. The control of these outbreaks was tempered by ongoing insecurity and 


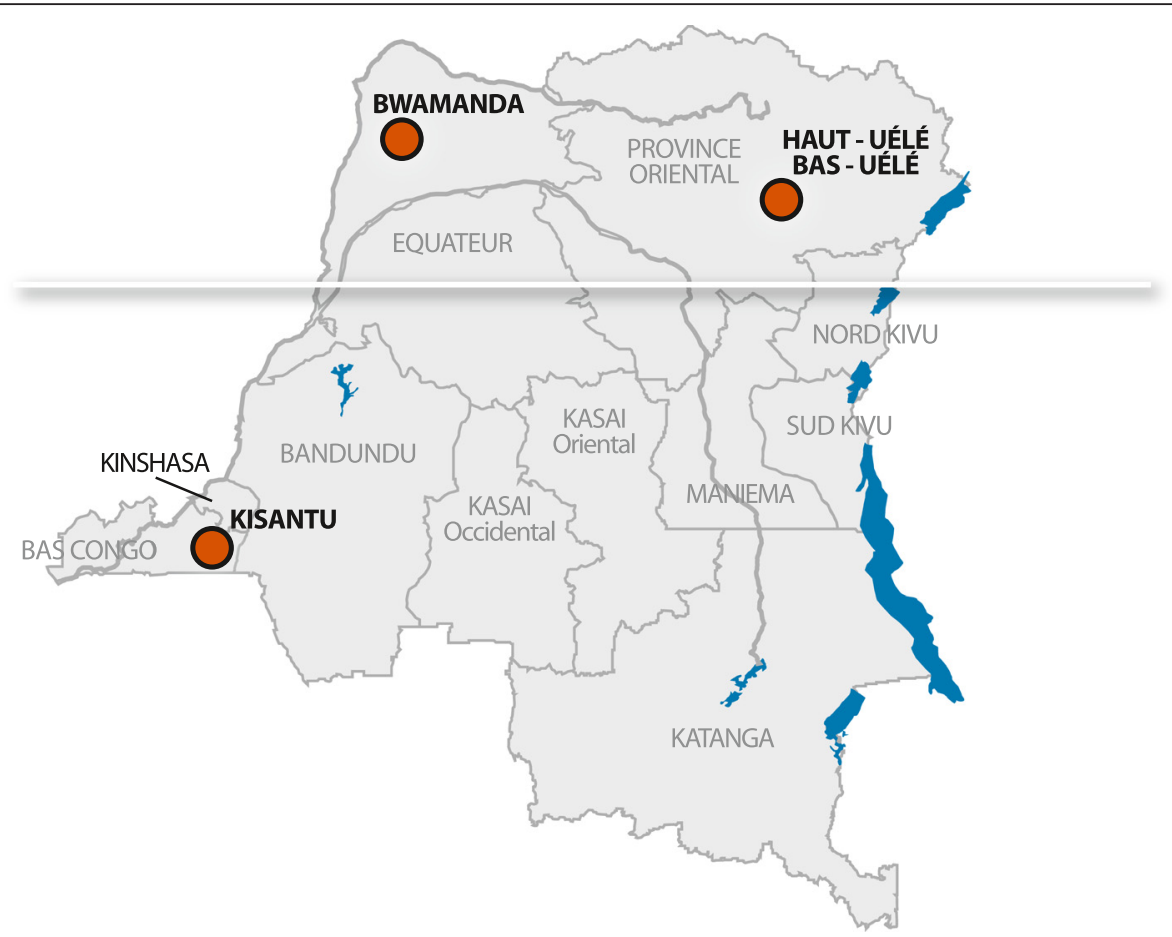

Fig. 1 Map of DRC with the three places recently affected by a microbiologically documented (epidemic) increase in Salmonella infections (i) Kisantu [15]; (ii) Bwamanda [16]; (iii) districts of Bas-Uélé and Haut-Uélé (present report). @ Institute of Tropical Medicine, Antwerp

poor transport infrastructure including the absence of paved roads.

\section{Blood culture surveillance network, CUKIS, Kisangani} The University Hospital of Kisangani (CUKIS), located in the capital of the Oriental Province, has basic laboratory infrastructure performing blood cultures and is participating in the microbiological surveillance network coordinated by the National Institute of Biomedical Research (INRB) in Kinshasa in collaboration with the Institute of Tropical Medicine (ITM) in Antwerp, Belgium. This network monitors the pathogens involved in bloodstream infections and their antibiotic resistance patterns at various sentinel sites in the DRC. CUKIS receives samples from hospitals and health centers in Kisangani and from more remote sites during outbreak investigations. CUKIS is located at $445 \mathrm{~km}$ from the $\mathrm{HZ}$ Wamba, $519 \mathrm{~km}$ from the HZ Pawa, $571 \mathrm{~km}$ from the HZ Isiro and $691 \mathrm{~km}$ from the HZ Poko.

Criteria for blood cultures sampling in patients $>28$ days of age were: (1) a body temperature of $\geq 38{ }^{\circ} \mathrm{C}$ or $\leq 35.5^{\circ} \mathrm{C}$; (2) suspicion of severe localized infections; or (3) suspicion of sepsis, typhoid fever, and severe malaria. Additional criteria for collection of blood specimens from women in the neonatal period were premature rupture of membranes, intrapartum fever, low Apgar score, or specific symptoms such as tachypnea, cyanosis, and lethargy. For children
( $\leq 14$ years old), $1-4 \mathrm{~mL}$ of blood was sampled into a pediatric blood culture vial (BacT/ALERT PF, bioMérieux, Marcy L'Etoile, France); for adults, $2 \times 10 \mathrm{~mL}$ of blood was inoculated into aerobic blood culture vials (BacT/ALERT FA, bioMérieux) and incubated at $35{ }^{\circ} \mathrm{C}$ for 7 days. Blood cultures were processed according to standard methods as described elsewhere [22, 23]. All isolates were stored in tubes of Trypticase Soya Agar (Oxoid, Basingstoke, UK) and shipped to ITM for confirmation and further identification.

\section{Serotyping and antibiotic susceptibility}

At ITM, isolates biochemically confirmed as Salmonella spp. were serotyped using commercial antisera (Sifin, Berlin, Germany). A selection of the isolates were sent to the National Reference Centre in Belgium for confirmation of serotyping by slide agglutination following the Kauffmann-White scheme [24]. Selection of these isolates was based on differences in year and location of collection.

Susceptibility testing of the isolates and interpretation of results were done according to the National Committee for Clinical Laboratory Standards (CLSI) guidelines as described elsewhere [23]. Antibiotic susceptibility tests were performed with the E-test macromethod (bioMérieux) (azithromycin, ciprofloxacin) or disk diffusion (Neo-Sensitabs, Rosco, Taastrup, Denmark) (all other 


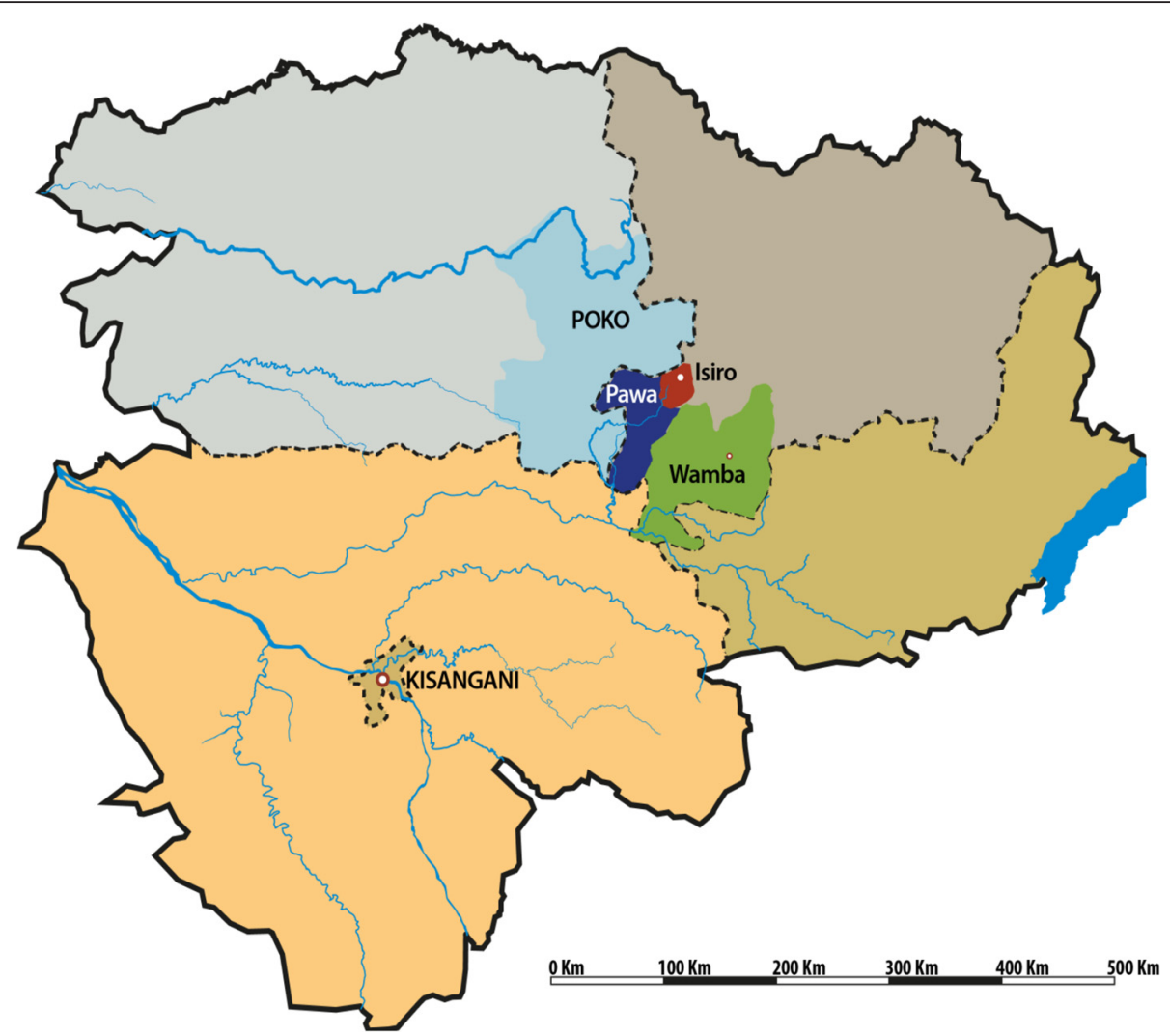

Fig. 2 The four health zones affected by the iNTS disease outbreak in 2012: Wamba and Isiro (Haut-Uélé) and Pawa and Poko (Bas-Uélé). (๑) Institute of Tropical Medicine, Antwerp

antibiotics). Multidrug resistance (MDR) was defined as co-resistance to all three first-line antibiotics ampicillin, chloramphenicol and trimethoprim-sulfamethoxazole [25].

The methods described apply equally to ongoing surveillance efforts and to the additional site visits done in 2012/2013.

\section{Determinations of mutations responsible for decreased ciprofloxacin susceptibility}

Molecular mechanisms of ciprofloxacin resistance were assessed by amplification and sequencing of the quinolone resistance determining regions (QRDRs) of the gyrA, gyrB, and parC genes as previously described [26]. The fragments were sequenced on an ABI 3130 sequencer. The presence of the plasmid-mediated quinolone resistance $q n r$ genes ( $q n r A, q n r B$ and $q n r S$ ) was determined using PCR [27].

\section{Multiple-locus variable-number of tandem repeats} analysis (MLVA) and assessment of clonality of Salmonella Enteritidis and Salmonella Typhimurium isolates

To assess the clonal relatedness among Salmonella Enteritidis and Salmonella Typhimurium isolates, MLVA analysis was performed on all isolates as previously described [28, 29]. For Salmonella Enteritidis, profiles were attributed based on the number of tandem repeats on five loci in the following order: SENTR7-, SENTR5-, SENTR6-, SENTR4- and SE3-. For Salmonella

Table 1 Demographic characteristics of the affected health zones (Pawa, Wamba, Poko and Isiro), Oriental Province, DRC

\begin{tabular}{llllcc}
\hline Health zones & $\begin{array}{l}\text { HGR capacity } \\
\text { (numbers of beds) }\end{array}$ & Surface $\left(\mathrm{km}^{2}\right)$ & Population (numbers) & $\begin{array}{l}\text { HIV prevalence } \\
\text { (Pregnant women, \%) }\end{array}$ & $\begin{array}{l}\text { Overall acute } \\
\text { malnutrition (\%) }\end{array}$ \\
\hline Pawa & 110 & 2415 & 157,657 & 4.7 & 12.2 \\
Wamba & 80 & 8203 & 130,473 & 6.2 & 10.4 \\
Poko & 100 & 10,876 & 168,567 & 5.7 & 13.3 \\
Isiro & 140 & 1646 & 208,658 & 4.5 & 7.6 \\
\hline
\end{tabular}


Typhimurium, profiles were attributed based on the number of tandem repeats on five loci in the following order: STTR9-, STTR5-, STTR6-, STTR10- and STTR3-. Simpson's index of diversity (D) and Shannon's indices of diversity $\left(\mathrm{H}^{\prime}\right)$ and equitability $(\mathrm{E})$ were calculated to evaluate the discriminatory power and evenness of distribution by using the Biodiversity Calculator [30].

\section{Clinical data}

Outreach visits to the HZs of Pawa, Isiro, Poko and Wamba were performed by an INRB team and a CUKIS collaborator (DF) in June/July 2012 and to the HZ of Pawa in the period October 2012-January 2013. During these and additional visits, clinical data from all children with a documented Salmonella bloodstream infection between 2009 and 2014 were retrieved from patient files. Data recorded included age, gender, weight and geographic origin, clinical presentation, diagnosis upon admission, number of blood transfusions, in-hospital outcome, Hb-value (Sahli method [31]) and parasitebased diagnosis of malaria (diagnosed either by microscopy or by rapid diagnostic test). Blood transfusions had been administered according to the criteria established by the national program of blood transfusion [32], i.e., $\mathrm{Hb}$ value below $6 \mathrm{~g} / \mathrm{dl}$ or signs of respiratory and circulatory failure. Anemia was defined as an $\mathrm{Hb}$ value $<11 \mathrm{~g} / \mathrm{dl}$ and severe anemia was defined as an $\mathrm{Hb}$-value $<5 \mathrm{~g} / \mathrm{dl}$. Nutritional status was assessed by the weight-for-age method which for children under 5 years compares the actual weight of the child to the referral charts indicating the ideal weight-for-age and defines low and very low weight-for-age $(\leq-2 \mathrm{SD}$ and $\leq-3$ SD from the reference respectively) [33]. No data on child height, HIV status or malaria density was available. The patient files of children who did not have a confirmed Salmonella bloodstream infection were not reviewed.

Data on hospital admissions and case-fatality rates were collected from the hospital registers and concerned all patients admitted to the pediatric ward. For the General Referral Hospital of Poko, blood transfusion files for the year 2011 could not be retrieved.

\section{Data on rainfall and climate}

Data of rainfall in the Oriental Province (2011-2013) were retrieved from the Global Information and Early Warning System from the Food and Agriculture organization of the United Nations [34].

\section{Data registration, statistical analysis}

Demographic, clinical and microbiological data were entered anonymously into an Excel database (Microsoft, Redmond, WA, USA). Only the first isolate for each patient was considered, isolates recovered from a second blood culture drawn within two weeks after the initial one were considered as duplicates, whereas isolates recovered from a repeat blood culture more than two weeks after the initial one were considered as recurrences (either relapse or repeat infections). Statistical analysis was done with Stata 12 (Stata Corp., College Station, TX, USA). P values and (adjusted) odds ratio's $(\mathrm{OR})$ were calculated by univariate and multivariate logistic regression analysis. When one group contained zero observations for a particular variable, the $\mathrm{p}$ value was calculated with a two tailed Fisher's exact test instead.

It was decided only to keep the categorical variables anemia and severe anemia rather than the continuous variable of hemoglobin level $(\mathrm{g} / \mathrm{dL})$, because of the limitations of the measurement (Sahli method) and because it seemed more relevant clinically. When multiple variables differed statistically between groups at a level of $\mathrm{P}<0.100$ and were also clinically relevant, they were further assessed in a multivariable logistic regression analysis. Diagnoses upon admission were not included in the multivariate analysis. To assess the influence of malaria positivity, initial analysis was done stratified for malaria positivity. Stepwise backward regression was used to eliminate variables that this did not retain their statistical significance. Likelihood ratio testing guided the selection of the final model. A p value of $<0.05$ was considered significant.

\section{Ethics statement}

The study was conducted according to the principles expressed in the Declaration of Helsinki. Ethical approval for the Microbiological Surveillance Study was granted by the Institutional Review Board of the ITM, the Ethics Committee of Antwerp University, and the Ministry of Health of the DRC. In view of the absence of an Ethical committee in Kisangani at the time of the study, written authorization to review the patient files was obtained from the Medical Inspector of the Oriental Province (Reference number: $\mathrm{N}^{0}$ 701/DPS/PERS/872/ 2012) who represents the highest health authority as well as from the Chief Medical Officer of the Haut-Uélé district (Reference number: 771/23/ISP/HU.O/2012). The retrieved clinical data were only accessible to the CUKIS collaborator (DF), who is a medical doctor, and were subsequently entered anonymously in an Excel database with restricted access.

\section{Results}

Data of hospital admissions, blood transfusions and malaria cases, 2011-2013

In Pawa and Wamba HZs, overall hospital admissions increased from March 2012 onwards, reaching a peak in May-June 2012 (Fig. 3a and b). In June 2012, numbers of 


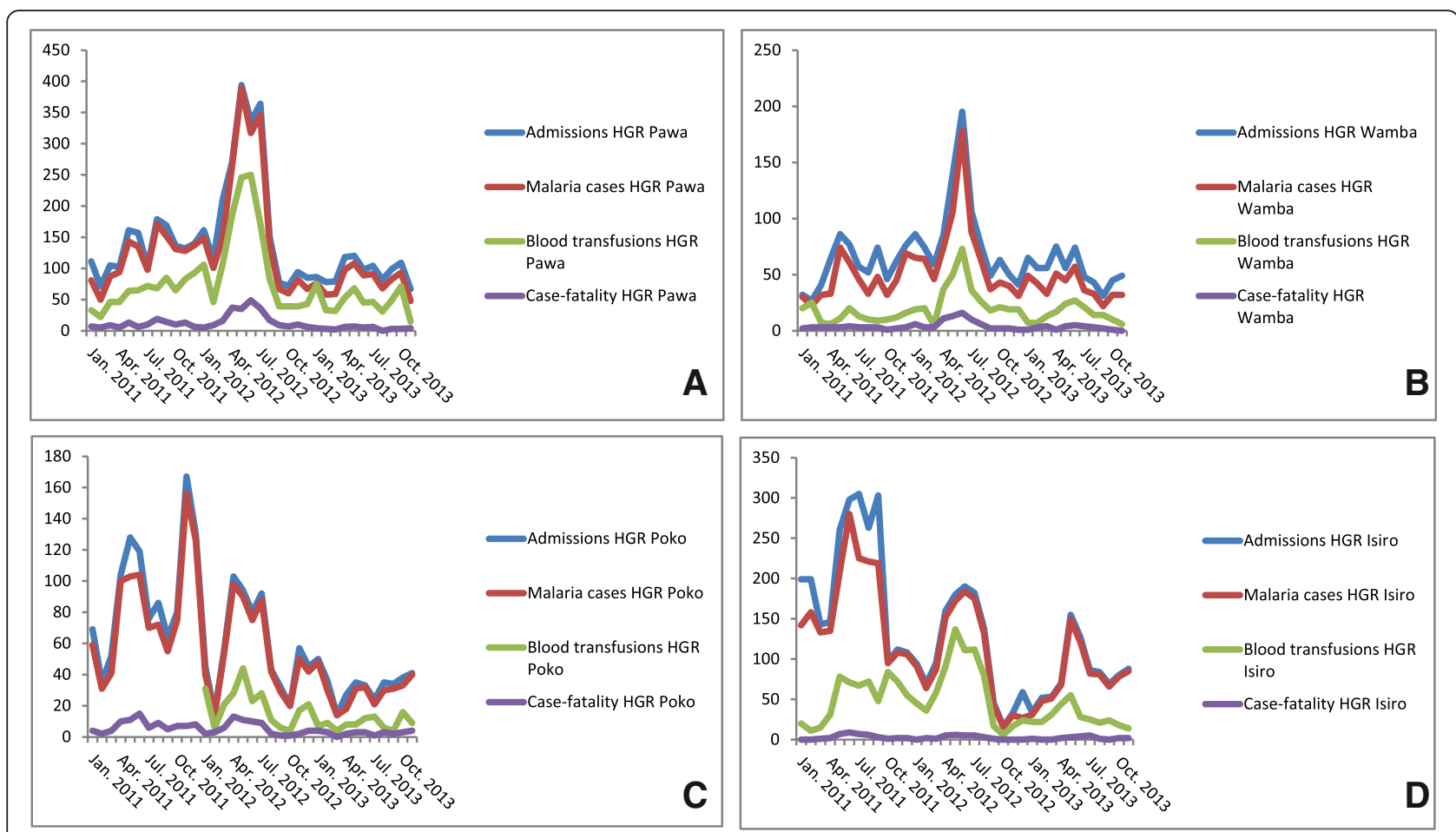

Fig. 3 Hospital admissions, malaria cases, blood transfusions and case-fatality rates (2011-2013) for a Pawa Health Zone Referral Hospital; b Wamba Health Zone Referral Hospital; c Poko Health Zone Referral Hospital; d Isiro Health Zone Referral Hospital. HGR = General Referal Hospital

overall admissions in Pawa were 2.6 times higher as compared to the average of the corresponding month during the years 2011 and 2013 (336 versus 128 respectively). The same was observed in Wamba (195 admissions in June 2012 vs. 77 and 74 in June 2011 and June 2013). The temporal evolution of numbers of malaria cases and blood transfusions aligned with those of the overall hospital admissions: in Pawa, 317 malaria cases were recorded in June 2012, versus an average of 112 for the other years (i.e., a nearly a three-fold increase); for blood transfusions, the difference was almost five-fold (250 versus 55). In Wamba a three-fold increase was seen for the number of malaria cases in June 2012 compared to the average of other years (178 vs. 59$)$, as well as for the number of blood transfusions (73 in June 2012 vs. 24 in other years). The number of case fatalities showed a similar evolution, with a total number of 49 fatalities in Pawa in June 2012 (i.e., an in-hospital case fatality rate of $14.6 \%(49 / 336)$ ) versus an average of 6 cases during the same month in the other years. In Wamba there were 16 fatalities in June 2012 vs. an average of 5 cases that month in other years. The HZs of Poko and Isiro showed a different trend, with a similar peak in June 2012, but with significant peaks in admission numbers and malaria cases already in 2011 (Fig. 3c and d). The increases of 2012 occurred approximately one month after the end of the dryer season, during which less rainfall had been observed compared to other years (Fig. 4).

\section{NTS infections in the Bas-Uélé and Haut-Uélé districts, 2012-2013}

In June 2012, at the request of the Ministry of Health, INRB staff did an outreach visit to all four affected HZs. Out of 87 blood cultures sampled, 18 (20.7 \%) grew clinically significant organisms (further referred to as pathogens), of which 16 (88.9\%) were NTS, with Salmonella Enteritidis and Salmonella Typhimurium accounting for nine and seven isolates respectively. Between October 2012 and January 2013 outreach visit to the HZ of Pawa allowed sampling of another 244 blood cultures, with 38 (15.6\%) pathogens, including Salmonella Enteritidis $(n=7)$ and Salmonella Typhimurium $(n=3) \quad(26.3 \%$ of pathogens). After Salmonella species, the most frequently isolated pathogens were Acinetobacter species and Staphylococcus aureus.

\section{Blood culture surveillance, CUKIS, Kisangani, Salmonella Typhi and NTS isolates (2009 - 2014)}

From May 2009 to May 2014, CUKIS processed 3467 blood cultures in total comprising $88.0 \%$ samples (3052/ 3467) from Kisangani district and $12.0 \%(415 / 3467)$ from the Haut-Uélé and Bas-Uélé districts, including samples from the outreach visits. In total, $11.4 \%$ 


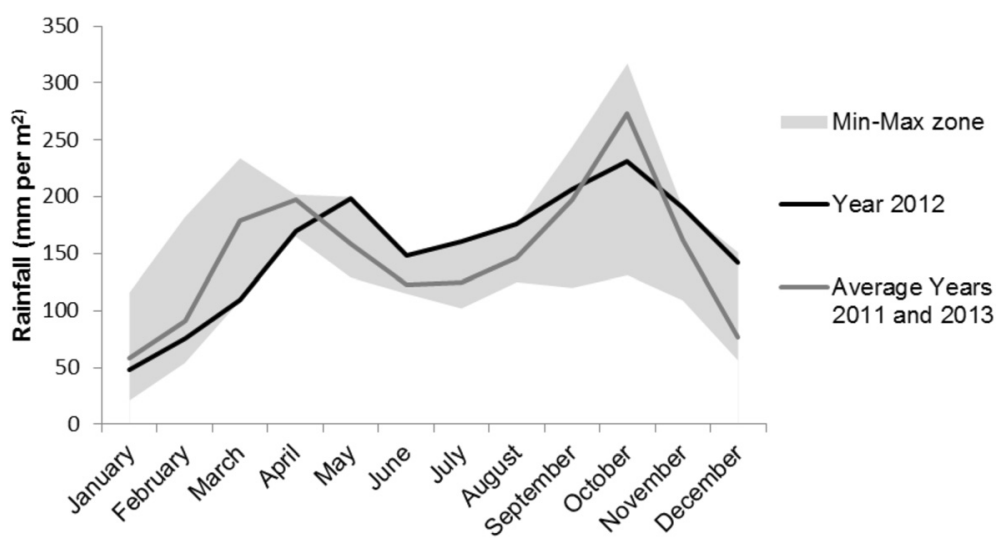

Fig. 4 Rainfall in the Oriental Province, DRC (2011-2013). Peak months of the outbreak were April 2012 to June 2012

(396/3467) blood cultures grew pathogens of which $34.1 \%(135 / 396)$ were Salmonella. The annual proportion varied between $25.0 \%$ (2009-2010) and $42.3 \%$ (2012). Salmonella were the most commonly isolated bacteria in both adults and children. Other frequent pathogens were Staphylococcus aureus and Klebsiella pneumoniae. Among the 135 Salmonella isolates, there were five duplicates (all grown within 14 days after the day of first sampling). Of the 130 first (unique) isolates, there were 17 Salmonella Typhi and 113 NTS isolates.

\section{NTS isolates Oriental Province, 2009-2014: serovars and clonality}

Salmonella Typhimurium and Salmonella Enteritidis were the most frequent serovars accounting for approximately half of NTS isolates each (47.8 and $49.6 \%$ out of 113 isolates respectively) (Table 2). In 2011 and 2012, Salmonella Enteritidis outnumbered Salmonella Typhimurium and vice versa during the years before and after. The other NTS serovars recovered were Salmonella Kisangani and Salmonella spI 1,4,12:-:1,2 (Salmonella group B).
MLVA analysis of the Salmonella Typhimurium isolates revealed heterogeneity with 23 different profiles across 54 isolates collected between 2009 and 2014 (Simpson's D = 0.95) (Additional file 1: Figure S1). The Salmonella Typhimurium isolates also showed an even distribution (Shannon's $H^{\prime}=2.90$ and $E=0.93$ ). Forty-four of the 54 Salmonella Typhimurium isolates were collected in the Tshopo district (where the provincial capital Kisangani is located), with three major profiles accounting for only 18 isolates $(40,9 \%)(2-6-11-8-0210 \quad(n=7), 2-5-10-8-0210$ $(n=6), 2-7-10-8-0210(n=5))$ in this district. Eight Salmonella Typhimurium isolates were collected in the Bas-Uéle district. Of these 8 , five belonged to the profile 2-8-12-8-0210, which was not isolated in the Tshopo district.

Multilocus variable-number tandem-repeat analysis (MLVA) of the 56 Salmonella Enteritidis isolates revealed less heterogeneity with 13 different profiles (Simpson's D = 0.71) (Additional file 2: Figure S2). Isolates were unevenly distributed over the subtypes, with two major profiles, i.e., 2-13-3-3-NA $(n=26)$ and 2-13-4-3NA $(n=15)$, accounting for $73.2 \%$ of the tested isolates (Shannon's H' $=1.64$ and $E=0.64$ ). Thirty-five out of the

Table 2 Distribution of Salmonella serovars among clinically significant organisms (CSO), Oriental Province, DRC, 2009-2014. Only first isolates were considered

\begin{tabular}{|c|c|c|c|c|c|c|}
\hline & 2009-2010* & 2011 & 2012 & 2013 & $2014^{* *}$ & Total \\
\hline & $(\mathrm{CSO}=92)$ & $(\mathrm{CSO}=83)$ & $(\mathrm{CSO}=78)$ & $(\mathrm{CSO}=89)$ & $(\mathrm{CSO}=54)$ & (\% of all Salmonella isolates) \\
\hline & n (\%) & n (\%) & n (\%) & n (\%) & n (\%) & \\
\hline Salmonella Typhi & 6 & 1 & 2 & 5 & 3 & $17(13.1)$ \\
\hline Salmonella Typhimurium & 9 & 6 & 13 & 14 & 12 & $54(41.5)$ \\
\hline Salmonella Enteritidis & 6 & 16 & 18 & 13 & 3 & $56(43.1)$ \\
\hline Other Salmonella serovars*** & 2 & 0 & 0 & 0 & 1 & $3(2.3)$ \\
\hline Total (\% of CSO) & $23(25.0)$ & $23(27.7)$ & $33(42.3)$ & $32(35.9)$ & $19(35.2)$ & $130(100)$ \\
\hline
\end{tabular}

* Data from 2009 and 2010 are grouped together as 2009 was considered as a pilot year (training and implementation)

** For 2014, data were completed till May 27th

*** Including Salmonella group B (one isolate) and Salmonella Kisangani (two isolates) 
56 Salmonella Enteritidis isolates were collected in the health district of Tshopo; 26 (74,3\%) of these 35 isolates had the major profile 2-13-3-3-NA. This profile was not isolated in the health districts of Bas-Uélé and Haut-Uélé. Of the 21 Salmonella Enteritidis isolates collected in the Bas-Uélé and Haut-Uélé districts between 2009 and 2014, 17 isolates were collected in the year 2012 of which 10 belonged to the second major profile 2-13-4-3-NA.

\section{Antibiotic resistance of Salmonella according to serovar}

Susceptibility testing was done for all isolates collected in the DRC between 2009 and 2014 as described elsewhere $[6,23]$. Here, we will describe the isolates collected in the Oriental Province during the period 20112014 $(n=107)$ as they were not discussed separately from isolates collected in other provinces of the DRC (Table 3; Additional file 3: Table S1).

In contrast to Salmonella Typhi, the majority of NTS (82/96, 85.4 \%) were MDR, and with slightly higher resistance rates among Salmonella Typhimurium compared to Salmonella Enteritidis. Two isolates, one Salmonella Typhi and one Salmonella Typhimurium (isolated in 2011 and 2013 respectively), showed decreased ciprofloxacin susceptibility. In the Salmonella Typhi isolate with a MIC-value of $0.19 \mu \mathrm{g} / \mathrm{ml}$ for ciprofloxacin, a gyrA resistance mutation (Asp87-Tyr/Glu133Gly) was detected; the Salmonella Typhimurium isolate with a MIC of $0.25 \mu \mathrm{g} / \mathrm{ml}$ for ciprofloxacin also showed a gyrA resistance mutation (Asp87-Asn). Both isolates were resistant to nalidixic acid.

For all NTS isolates combined, MIC50 and MIC90 values for ciprofloxacin were $0.012 \mu \mathrm{g} / \mathrm{ml}$ and $0.047 \mu \mathrm{g} / \mathrm{ml}$ respectively. The MIC50 value for ciprofloxacin of these isolates was $0.012 \mu \mathrm{g} / \mathrm{ml}$ in the period 2011-2012 and $0.016 \mu \mathrm{g} / \mathrm{ml}$ in the period 2013-2014 while the MIC90

Table 3 Antibiotic resistance rates of 107 invasive Salmonella isolates, Oriental Province, DRC, 2011-2014

\begin{tabular}{lcccc}
\hline & $\begin{array}{l}\text { Salmonella } \\
\text { Typhi } \\
n=11(\%)\end{array}$ & $\begin{array}{l}\text { Salmonella } \\
\text { Enteritidis } \\
n=50(\%)\end{array}$ & $\begin{array}{l}\text { Salmonella } \\
\text { Typhimurium } \\
n=45(\%)\end{array}$ & $\begin{array}{c}\text { Other } \\
\text { NTS }^{\mathrm{a}}\end{array}$ \\
$n=1$
\end{tabular}

TMP-SMX trimethoprim-sulfamethoxazole, MDR multi-drug resistant, DCS decreased ciprofloxacin susceptibility

asalmonella Group B value increased from 0.016 to $0.047 \mu \mathrm{g} / \mathrm{ml}$. All isolates were susceptible to ceftriaxone and azithromycin.

\section{Clinical data of children with invasive salmonellosis according to serovar (2009-2014)}

Clinical charts of children with iNTS diagnosed through ongoing surveillance, as well as during the additional site visits in 2012-2013, were reviewed.

A total of $91 / 117(77.8 \%)$ children with invasive salmonellosis were $<5$ years old. As expected, the median age for children with iNTS disease (24 months (IQR 12-36)) was significantly lower as compared to children infected with Salmonella Typhi (96 months (IQR 48-123); $p=0.000$ ).

For 88 out of $124(71.0 \%)$ children with invasive salmonellosis clinical files were available (Tables 4 and 5). There was a significant difference in diagnosis upon admission between iNTS disease and Salmonella Typhi cases; the diagnosis of typhoid fever was raised in $68.8 \%$ of children with confirmed Salmonella Typhi infection compared to $18.5 \%$ of children with iNTS disease $(p=$ $0.000)$. This was in line with differences in the clinical presentation: in particular, dyspnea and pallor tended to occur more frequently among children with iNTS disease compared to children with Salmonella Typhi infection ( $46.7 \%$ vs. $23.1 \%$ and $76 \%$ vs. $38.5 \%(p=0.010)$ respectively), while vomiting tended to be less frequent (45.3\% vs. $69.2 \%$ ). Bivariate analysis also showed significant differences in malaria positivity $(69.3 \%$ vs. $30.8 \%$; $p=$ 0.012 ), anemia (98.6 \% vs. $76.9 \%$; $p=0.010)$, and rate of blood transfusions ( $65.3 \%$ vs. $30.8 \% ; p=0.026$ ) between the children with iNTS disease vs. the children with a Salmonella Typhi infection respectively. We included these variables in the multivariate analysis and initially stratified for malaria positivity. In both groups (malaria positives vs. malaria negatives), age (months) was the only variable that remained significant with an OR similar in both groups. We therefore decided to include malaria positivity as a variable in the multivariable analysis. Likelihood ratio testing indicated that the best model included the variables age (adjusted OR 0.95 (95\% CI 0.93-0.97)), malaria positivity (adjusted OR 6.31 (95\% CI 0.99-39.95) and pallor (adjusted OR 5.99 (95\% CI 0.95-37.77). Age remained significant $(p=0.000)$ indicating that younger age is associated with non-typhoidal Salmonella infection while malaria positivity and pallor were borderline significant $(p=$ 0.050 and $p=0.057$ respectively).

Mortality in children with iNTS disease was $13.3 \%$ $(10 / 75)$ compared to $7.7 \%(1 / 13)$ of the children with a Salmonella Typhi infection; this did not differ significantly. Seventy per cent (7/10) of deaths due to iNTS disease occurred within the first $24 \mathrm{~h}$ after admission.

The median age of children with a Salmonella Typhimurium bloodstream infection (18 months (IQR 12-30)) 
Table 4 Demographic, clinical and laboratory data of children with a Salmonella Typhi or non-typhoidal Salmonella infection, Oriental Province, DRC, 2009-2014. Except for age, data represent numbers (\%)

\begin{tabular}{|c|c|c|c|c|c|c|c|c|}
\hline \multirow[b]{2}{*}{ Characteristics } & \multicolumn{2}{|c|}{ Salmonella Typhi } & \multicolumn{2}{|c|}{ Non-typhoidal Salmonella } & \multicolumn{2}{|l|}{ Bivariate analysis } & \multicolumn{2}{|c|}{ Multivariate analysis } \\
\hline & $\mathrm{N}$ & Yes (\%) & $\mathrm{N}$ & Yes (\%) & $\mathrm{OR}(\mathrm{Cl})$ & p-value & $\mathrm{aOR}(\mathrm{Cl})$ & p-value \\
\hline \multicolumn{9}{|l|}{ Demographics } \\
\hline Median age, months (IQR) & 16 & $96(48-123)$ & 101 & $24(12-36)$ & $0.96(0.94-0.97)$ & 0.000 & $0.95(0.93-0.97)$ & 0.000 \\
\hline Male gender & 16 & $7(43.8)$ & 108 & $63(58.3)$ & $1.8(0.62-5.19)$ & 0.277 & & \\
\hline \multicolumn{9}{|l|}{ Nutritional data (age <60 months) } \\
\hline Low weight-for-age & 3 & $0(0.0)$ & 69 & $14(20.3)$ & * & $1.000^{\mathrm{b}}$ & & \\
\hline Very low weight-for-age & 3 & $0(0.0)$ & 69 & $11(15.9)$ & * & $1.000^{\mathrm{b}}$ & & \\
\hline \multicolumn{9}{|l|}{ Symptoms at admission } \\
\hline Vomiting & 13 & $9(69.2)$ & 75 & $34(45.3)$ & $0.36(0.10-1.30)$ & 0.121 & & \\
\hline Diarrhea & 13 & $2(15.4)$ & 75 & $21(28.0)$ & $2.13(0.43-10.47)$ & 0.348 & & \\
\hline Cough & 13 & $8(61.5)$ & 75 & $40(53.3)$ & $0.71(0.21-2.38)$ & 0.584 & & \\
\hline Dyspnea & 13 & $3(23.1)$ & 75 & $35(46.7)$ & $2.91(0.74-11.45)$ & 0.125 & & \\
\hline Lethargy & 13 & $9(69.2)$ & 75 & $44(58.7)$ & $0.63(0.17-2.23)$ & 0.475 & & \\
\hline Coma & 13 & $0(0.0)$ & 75 & $1(1.3)$ & * & $1.000^{b}$ & & \\
\hline Seizures & 13 & $0(0.0)$ & 75 & $7(9.3)$ & * & $0.588^{b}$ & & \\
\hline Pallor & 13 & $5(38.5)$ & 75 & $57(76.0)$ & $5.06(1.47-17.44)$ & 0.010 & $5.99(0.95-37.77)$ & 0.057 \\
\hline Splenomegaly & 13 & $3(23.1)$ & 69 & $18(26.1)$ & $1.17(0.29-4.75)$ & 0.820 & & \\
\hline Hepatomegaly & 13 & $0(0.0)$ & 69 & $13(18.8)$ & * & $0.115^{\mathrm{b}}$ & & \\
\hline Antibiotic exposure $<2$ weeks & 15 & $5(33.3)$ & 95 & $34(35.8)$ & $1.11(0.35-3.52)$ & 0.853 & & \\
\hline \multicolumn{9}{|l|}{ Diagnosis upon admission ${ }^{c}$} \\
\hline Malaria & 16 & $5(31.3)$ & 107 & $58(54.2)$ & $2.60(0.84-8.00)$ & 0.088 & & \\
\hline Pneumonia & 16 & $1(6.3)$ & 107 & $17(15.7)$ & $2.83(0.35-22.89)$ & 0.329 & & \\
\hline Typhoid fever & 16 & $11(68.8)$ & 107 & $20(18.7)$ & $0.10(0.03-0.33)$ & 0.000 & & \\
\hline Septicemia & 16 & $0(0.0)$ & 107 & $13(12.1)$ & $*$ & $0.214^{b}$ & & \\
\hline Urinary tract infection & 16 & $1(6.3)$ & 107 & $7(6.5)$ & $1.15(0.12-9.14)$ & 0.965 & & \\
\hline Gastro-intestinal tract infection & 16 & $0(0.0)$ & 107 & $4(3.7)$ & * & $1.000^{\mathrm{b}}$ & & \\
\hline Meningitis & 16 & $1(6.3)$ & 107 & $8(7.4)$ & $1.2(0.13-10.28)$ & 0.861 & & \\
\hline Other diagnosis & 16 & $5(31.3)$ & 107 & $13(12.0)$ & $0.30(0.09-1.00)$ & 0.051 & & \\
\hline \multicolumn{9}{|l|}{ Laboratory data } \\
\hline Malaria diagnosis ${ }^{\mathrm{a}}$ & 13 & $4(30.8)$ & 75 & $52(69.3)$ & $5.08(1.42-18.22)$ & 0.012 & $6.31(0.99-39.95)$ & 0.050 \\
\hline Anemia $(\mathrm{Hb}<11 \mathrm{~g} / \mathrm{dl})$ & 13 & $10(76.9)$ & 74 & 73 (98.6) & $21.9(2.07-231.40)$ & 0.010 & & \\
\hline Severe anemia $(\mathrm{Hb}<5 \mathrm{~g} / \mathrm{dl})$ & 13 & $1(7.7)$ & 74 & $22(29.7)$ & $5.07(0.62-41.45)$ & 0.129 & & \\
\hline \multicolumn{9}{|l|}{ Transfusion/Outcome } \\
\hline Blood transfusion & 13 & $4(30.8)$ & 75 & $49(65.3)$ & $4.24(1.19-15.10)$ & 0.026 & & \\
\hline Died in hospital & 13 & $1(7.7)$ & 75 & $10(13.3)$ & $1.84(0.21-15.78)$ & 0.575 & & \\
\hline
\end{tabular}

OR Odd's'ratio, $a O R$ adjusted Odd's ratio

"Predicts failure perfectly

a Malaria diagnosis as confirmed by either thick blood film microscopy or rapid diagnostic test

${ }^{\mathrm{b}}$ Fisher exact test was used to calculate $\mathrm{p}$ value (other variables: logistic regression)

'Diagnoses upon admission were not included in the multivariate analysis

was lower compared to those with a Salmonella Enteritidis bloodstream infection (24 months (IQR 12-48)) but this difference was not statistically significant $(p=0.128)$. For all iNTS disease cases combined, malaria represented the most frequent diagnosis upon admission (made in nearly half of cases) followed by, in rank of decreasing frequency, typhoid fever, pneumonia and septicemia. Malaria was suspected more often in children with a Salmonella Enteritidis infection compared to children with a Salmonella Typhimurium infection $(65,4 \%$ vs. $44,2 \% ; p=0.032)$. 
Table 5 Demographic, clinical and laboratory data of children with a Salmonella Typhimurium or Salmonella Enteritidis infection, Oriental Province, DRC, 2009-2014. Except for age, data represent numbers (\%)

\begin{tabular}{|c|c|c|c|c|c|c|}
\hline \multirow[b]{2}{*}{ Characteristics } & \multicolumn{2}{|c|}{$\underline{\text { Salmonella Typhimurium }}$} & \multicolumn{2}{|c|}{ Salmonella Enteritidis } & \multicolumn{2}{|l|}{ Bivariate analysis } \\
\hline & $\mathrm{N}$ & Yes (\%) & $\mathrm{N}$ & Yes (\%) & $\mathrm{OR}(\mathrm{Cl})$ & p-value \\
\hline \multicolumn{7}{|l|}{ Demographics } \\
\hline Median age, months (IQR) & 51 & $18(12-30)$ & 47 & $24(12-48)$ & $1.00(0.99-1.02)$ & 0.275 \\
\hline Male gender & 53 & $27(50.9)$ & 52 & $33(63.5)$ & $1.66(0.77-3.56)$ & 0.188 \\
\hline \multicolumn{7}{|l|}{ Nutritional data (age <60 months) } \\
\hline Low weight-for-age & 38 & $8(21.1)$ & 30 & $6(20.0)$ & $0.93(0.28-3.07)$ & 0.915 \\
\hline Very low weight-for-age & 38 & $6(15.8)$ & 30 & $5(16.7)$ & $1.06(0.29-3.90)$ & 0.922 \\
\hline \multicolumn{7}{|l|}{ Symptoms at admission } \\
\hline Vomiting & 40 & $17(42.5)$ & 33 & $16(48.5)$ & $1.27(0.50-3.21)$ & 0.609 \\
\hline Diarrhea & 40 & $9(22.5)$ & 33 & $12(36.4)$ & $1.96(0.70-5.49)$ & 0.196 \\
\hline Cough & 40 & $25(62.5)$ & 33 & $15(45.5)$ & $0.5(0.19-1.27)$ & 0.147 \\
\hline Dyspnea & 40 & $19(47.5)$ & 33 & $16(48.5)$ & $1.04(0.41-2.61)$ & 0.933 \\
\hline Lethargy & 40 & $22(55.0)$ & 33 & $21(63.6)$ & $1.43(0.55-3.67)$ & 0.456 \\
\hline Coma & 40 & $1(2.5)$ & 33 & $0(0.0)$ & * & $1.000^{\mathrm{b}}$ \\
\hline Seizures & 40 & $7(17.5)$ & 33 & $0(0.0)$ & * & $0.014^{b}$ \\
\hline Pallor & 40 & $29(72.5)$ & 33 & $28(84.8)$ & $2.12(0.65-6.89)$ & 0.210 \\
\hline Splenomegaly & 37 & $7(18.9)$ & 30 & $11(36.7)$ & $2.48(0.81-7.51)$ & 0.108 \\
\hline Hepatomegaly & 37 & $6(16.2)$ & 30 & $7(23.3)$ & $1.57(0.46-5.30)$ & 0.466 \\
\hline Antibiotic exposure $<2$ week & 49 & 15 (30.6) & 44 & $19(43.2)$ & $1.72(0.73-4.03)$ & 0.211 \\
\hline \multicolumn{7}{|l|}{ Diagnosis upon admission } \\
\hline Malaria & 52 & $23(44.2)$ & 52 & $34(65.4)$ & $2.38(1.07-5.25)$ & 0.032 \\
\hline Pneumonia & 52 & $9(17.3)$ & 52 & $7(13.5)$ & $0.74(0.25-2.17)$ & 0.588 \\
\hline Typhoid fever & 52 & $11(21.2)$ & 52 & $8(15.4)$ & $0.67(0.24-1.85)$ & 0.448 \\
\hline Septicemia & 52 & $5(9.6)$ & 52 & $8(15.4)$ & $1.70(0.51-5.62)$ & 0.378 \\
\hline Urinary tract infection & 52 & $1(1.9)$ & 52 & $4(7.7)$ & $4.25(0.45-39.38)$ & 0.203 \\
\hline Gastro-intestinal tract infection & 52 & $3(5.7)$ & 52 & $1(1.9)$ & $0.32(0.03-3.18)$ & 0.331 \\
\hline Meningitis & 52 & $4(7.7)$ & 52 & $4(7.7)$ & $1.00(0.23-4.23)$ & 1.000 \\
\hline Other diagnosis & 52 & $6(11.5)$ & 52 & $7(13.5)$ & $1.19(0.37-3.82)$ & 0.767 \\
\hline \multicolumn{7}{|l|}{ Laboratory data } \\
\hline Malaria diagnosis $^{\mathrm{a}}$ & 40 & $25(62.5)$ & 33 & $27(81.8)$ & $2.7(0.90-8.04)$ & 0.075 \\
\hline Anemia $(\mathrm{Hb}<11 \mathrm{~g} / \mathrm{dl})$ & 39 & $38(97.4)$ & 33 & $33(100)$ & * & $1.000^{b}$ \\
\hline Severe anemia $(\mathrm{Hb}<5 \mathrm{~g} / \mathrm{dl})$ & 39 & $10(25.6)$ & 33 & $12(36.4)$ & $1.65(0.60-4.54)$ & 0.327 \\
\hline \multicolumn{7}{|l|}{ Transfusion/Outcome } \\
\hline Blood transfusion & 40 & $25(62.5)$ & 33 & 24 (70.6) & $1.60(0.58-4.34)$ & 0.356 \\
\hline Died in hospital & 40 & $7(17.5)$ & 33 & $3(9.1)$ & $0.47(0.11-1.98)$ & 0.306 \\
\hline
\end{tabular}

OR Odd's'ratio

"Predicts failure perfectly

${ }^{a}$ Malaria diagnosis as confirmed by either thick blood film microscopy or rapid diagnostic test

${ }^{b}$ Fisher exact test was used to calculate $\mathrm{p}$ value (other variables: logistic regression)

The most common presenting symptom in children with iNTS disease was pallor, observed in $76 \%$ of all children, followed by lethargy, cough, dyspnea, and vomiting. Diarrhea was reported in only $28 \%$ of the children. The spectrum of symptoms did not differ between the different serovars except for seizures which were only observed in the Salmonella Typhimurium group (7 cases only; $p=0.014$ ).

Laboratory confirmed $P$. falciparum malaria was recorded in nearly $70 \%$ of all children with iNTS disease; this proportion tended to be higher among the Salmonella Enteritidis group compared to the 
Salmonella Typhimurium group (62.5 \% vs. $81.8 \%$; $p=0.075)$.

There was no significant difference in mortality between children infected with Salmonella Enteritidis and Salmonella Typhimurium.

Overall, 35,5 \% (39/110) of patients with a Salmonella bloodstream infection reported previous antibiotic exposure.

\section{Discussion}

The present study describes the clinical, microbiological and molecular characteristics of invasive Salmonella infections in the Oriental Province during the surveillance period 2009-2014, including data from outreach visits to more remote areas in the province during an epidemic increase of malaria in 2012.

During the surveillance period, the proportion of Salmonella among pathogens from blood cultures varied between 25.0 and $42.3 \%$, which is consistent with other studies from sub-Saharan Africa [2, 35-37]. There was a predominance of serovars Salmonella Typhimurium and Salmonella Enteritidis that were isolated in equal numbers but with proportions varying over time which is also in line with other studies from sub-Saharan Africa $[1,38,39]$. There was a particular high percentage (88.9 \%) of Salmonella among the pathogens isolated from blood cultures in the health zones affected by the epidemic increase in malaria in June 2012. During subsequent outreach visits between October 2012-January 2013 (period after the epidemic increase in malaria), this proportion subsequently decreased to only $26.3 \%$. The latter percentage is more in line with the averages at the provincial level, and suggests that the epidemic increase of malaria was associated with a concomitant increase in Salmonella bloodstream infections. Possibly, the data represented here are an underestimation of the actual number of patients co-infected with malaria and NTS. Other studies have shown that case fatalities are higher for children who are co-infected with NTS compared to those infected with malaria alone and, in the present study, part of them might have died before reaching the hospital [40].

The clinical presentation of iNTS disease in this study was dominated by pallor, lethargy, gastro-intestinal and respiratory symptoms. A concomitant infection with pneumococci has been forwarded to explain the prevalence of respiratory symptoms during iNTS disease but the dyspnea could also be related to the high prevalence of anemia among children with iNTS disease or to the acidosis due to sepsis in general [2]. There were no statistically significant differences between the characteristics of Salmonella Typhimurium and Salmonella Enteritidis bloodstream infections except for occurrence of seizures but this was probably affected by a small sample size.
Children with a Salmonella Enteritidis bloodstream infection tended to suffer from concomitant malaria more frequently compared to those infected with Salmonella Typhimurium. We are not aware of other studies that pointed to a particular association of Salmonella Enteritidis with concurrent malaria infection. More extensive and prospective research is needed to evaluate this finding further.

Overall, more than two-thirds of the children with iNTS disease tested positive for $P$. falciparum malaria which was also previously reported from Bwamanda in the DRC [16]. It is known that P. falciparum malaria predisposes to iNTS disease [7-9, 41, 42] and several recent studies have revealed probable underlying mechanisms [7, 43-45]. In the case of the Oriental Province, no clear reason for the upsurge in malaria was identified. The fact that during the end of the dry season less than average rainfall was observed might have played a role in the increase of malaria infections. Also, the influx of persons from conflict areas in the Kivu provinces, that have lower exposure to malaria compared to the Oriental Province (annually averaged infection prevalence of malaria in $2-10$ year olds $(<5 \%)$ compared to $>40 \%$ respectively), might have contributed to the increase [46].

Besides malaria, other known risk factors for contracting iNTS disease are HIV, sickle cell anemia and malnutrition. It is estimated that $12 \%$ of the hospitalized children in the DRC have sickle cell anemia [47]. For HIV, rates among pregnant women in the different districts of the Oriental Province varied between 4.5 and $6.2 \%$ (Table 1). In the study cohort described, severe malnutrition was not very common.

As there was also clinical data available for 13 children with a Salmonella Typhi infection, differences with iNTS cases could be explored. Vomiting was more prevalent among children infected with Salmonella Typhi compared to those with iNTS disease. Mtove et al. (2010) had similar observations in a study from rural Tanzania (71.3\% of Salmonella Typhi cases vs. $53.4 \%$ of iNTS disease cases) [11]. In bivariate analysis, significantly less children infected with Salmonella Typhi had pallor, anemia, need for blood transfusion and were less frequently tested positive for malaria compared to the children with iNTS. In the study of Mtove et al. (2010) children with Salmonella Typhi infections did not show pallor or severe anemia but children with iNTS did in respectively 62.2 and $40.0 \%$ of the cases [11]. Other studies have also described high numbers of patients with iNTS disease receiving blood transfusions [39, 42]. The final regression model included the variables age, malaria positivity and pallor, but only younger age remained significantly associated with non-typhoidal Salmonella infection. These results should be interpreted with caution however given the small sample sizes. 
Unlike the epidemic increase of iNTS in Kisantu which was only identified upon retrospect -the Oriental Province health authorities were alerted to the possibility of an increase in iNTS disease in concurrence with the observed increase in malaria cases. As a consequence, empirical antibiotic treatment was installed early on in the epidemic and this may have accounted for the relatively low case-fatality among the microbiologically confirmed iNTS cases in our cohort. This rate is similar to that found during the outbreak in Bwamanda [16] but lower to that found in Kisantu (23.0\%) [15], the latter being more in line with case fatality rates more frequently reported for iNTS $[2,4]$. In addition, given the large surface and geographical inaccessibility of the affected districts in the Oriental Province, with a swamp environment and absence of paved roads, many children are expected to have died at home or during their way to the health care center or hospital. The fact that most documented in-hospital deaths occurred within $24 \mathrm{~h}$ after admission supports this hypothesis.

Antibiotic susceptibility testing showed high MDR rates among NTS isolates; only two Salmonella isolates showed decreased ciprofloxacin susceptibility. However, MIC50 and MIC90 values for ciprofloxacin were higher in the period 2013-2014 compared to the period 20112012 which is a worrisome trend.

MLVA profiles of Salmonella Enteritidis isolates showed that two closely related profiles accounted for $73.2 \%$ of all the tested isolates. The two major profiles only differed with one tandem repeat at one single locus suggesting very close relatedness [28]. MLVA profiles of the Salmonella Enteritidis isolates implicated in the epidemic increases in Kisantu and Bwamanda also showed very close relatedness [28]. Whole genome sequencing of these isolates could provide more insight in the clonality of Salmonella Enteritidis associated with iNTS disease.

Salmonella Typhimurium isolates were more heterogeneous than Salmonella Enteritidis isolates with the two most common MLVA profiles accounting for only $24.1 \%$ of all tested isolates. The major profile isolated from the Haut-Uélé district differed by 1-2 tandem repeats at two loci compared to the major profiles isolated in the Tshopo district. Previous reports on epidemic increases of NTS infections in the DRC did not consider the MLVA profiles for Salmonella Typhimurium. A previous study did assess the population structure of 180 Salmonella Typhimurium isolates collected in the DRC between 2007 and 2011 with CRISPOL typing and found that $>96 \%$ belonged to the CT28 group [48]. This group has a strong association with Salmonella Typhimurium multilocus sequence type (ST) 313 which is associated with bloodstream infections and high rates of antimicrobial drug resistance. Also in other Sub-Saharan countries, the majority of isolates are found to be ST 313 [49]. Future research will include genomic sequencing of Salmonella Typhimurium isolates from different parts of the DRC, including isolates from the Oriental Province, Kisantu and Bwamanda.

The present study had several limitations, mostly owing to its retrospective nature. First, the outreach visits to the HZs were - due to logistic and administrative constraints - organized at a period considerably after the first alerts of increased admissions and malaria cases. Next, there was no clearly defined population size denominator, precluding calculation of population-based incidence rates. In addition, it could not be ascertained how many children died at home or on their way to the hospital, precluding accurate estimates of case-fatality rates. Furthermore, not all isolates, patients' files and hospital registers were available for analysis. Other difficulties included accessibility of the affected districts requiring for instance transport of samples by motorbikes on deteriorated roads and subsequent shipment by a small airplane carrier (at a distance of $350 \mathrm{~km}$ ) to CUKIS, the single diagnostic microbiology laboratory in the Oriental Province.

There were also limitations with regard to laboratory data: no distinction could be made between recent (microscopy negative but rapid diagnostic test positive) versus current malaria (microscopy positive), and the quality of malaria diagnosis probably differed per medical center. The limitations of measuring hemoglobin levels with the Sahli method should be kept in mind especially as no quality control for harmonization was performed.

Finally, it was not possible to compare the clinical characteristics of children with iNTS disease to the other children admitted, precluding a control group. Also, sampling of the affected health districts was mainly restricted to the year 2012, not allowing for comparisons with years before and after.

Sampling during the surveillance period was however constant over time and directed by a set of clinical indications, and clear comparisons with other years could be made. We believe this was also the case for the hospital registers providing compiled data about malaria diagnosis and blood transfusions.

Despite the limitations, this study offers valuable data on a neglected disease from an under investigated area collected during a time of great political instability. Previous articles have highlighted the need for studies that combine epidemiological, microbiological and clinical data on iNTS disease [5]. Last, the study allowed for a comparison between the most dominant NTS serovars. 
Future studies should estimate reliable population incidence rates. The development of an (antigen based) rapid diagnostic test would be highly welcome as a tool for diagnosis in low resource settings where microbiological facilities are often absent. More research is also needed on differences between the clinical presentation and outcome of both Salmonella Typhimurium and Salmonella Enteritidis serovars. Whole genome sequencing of NTS isolates from different areas in the DRC, especially those related to local increases in infections, would provide valuable insights. It is obvious that continued blood culture surveillance is imperative to monitor the proportions and distributions of NTS serovars as well as their antibiotic resistance patterns. Last, efforts to control the increase in malaria in this region should be intensified.

\section{Conclusion}

The present observations confirm NTS as a leading cause of bloodstream infection in an area holoendemic for P. falciparum malaria. A sudden increase in hospital admissions among children $<5$ years old, associated with a laboratory-confirmed diagnosis of $P$. falciparum malaria needs to raise the suspicion of a concomitant increase in NTS infections, particularly if associated with an increase in blood transfusions and case-fatality.

\section{Additional files}

Additional file 1: Figure S1. Minimum-spanning tree analysis of MLVA (multiple-locus variable-number tandem-repeats analysis) data of 54 Salmonella Typhimurium isolates, Oriental Province, DRC, 2009-2014. Each circle in this figure displays a unique MLVA type, with different colours indicating the year of collection of isolates. (PDF $308 \mathrm{~kb}$ )

Additional file 2: Figure S2. Minimum-spanning tree analysis of MLVA (multiple-locus variable-number tandem-repeats analysis) data of 56 Salmonella Enteritidis isolates, Oriental Province, DRC, 2009-2014. Each circle in this figure displays a unique MLVA type, with different colours indicating the year of collection of isolates. (PDF $112 \mathrm{~kb}$ )

Additional file 3: Table S1. Dataset of isolates collected in the Oriental Province, DRC between 2009 and 2014 that were used for this paper. DCS = Decreased Ciprofloxacin Susceptibility; R = Resistance; ZOl: Zone of inhibition. (XLSX $17 \mathrm{~kb})$

\section{Abbreviations \\ (a)OR, (adjusted) Odd's ratio; CLSI, National Committee for Clinical Laboratory Standards; CSO, clinically significant organisms; CUKIS, University Hospital of Kisangani; DCS, decreased ciprofloxacin susceptibility; DF, Dadi Falay; DRC, Democratic Republic of the Congo; HGR, General Referral Hospital; $\mathrm{HZ}$, health zone; INRB, National Institute of Biomedical Research; iNTS, invasive non- typhoid salmonella; IQR, interquartile range; ITM, Institute of Tropical Medi- cine; MDR, multidrug resistance; MLVA, multilocus variable-number tandem- repeat analysis; NTS, non-typhoidal Salmonella; RDT, rapid diagnostic test;} TMP-SMX, trimethoprim-sulfamethoxazole

\section{Acknowledgements}

The authors would like to thank Marleen Verlinden and Brigitte Mapendo Sibkasibka for technical assistance, and Jorgen Stassijns and the reviewers for their valuable comments that improved the quality of the manuscript. The authors would also like to thank Achilleas Tsoumanis, Epco Hasker and Saskia Decuypere for statistical support.

\section{Funding}

Several funders contributed to the research underlying this manuscript including the University of Leuven, the Flemish Interuniversity Council (VLIR-UOS), and the Belgian Directorate General of Development Cooperation (Ministry of Development Cooperation). One of the authors (Marie-France Phoba) received a scholarship of the Foundation Marguerite-Marie Delacroix, Tienen, Belgium.

A second author (Dadi Falay) was supported the Belgian Directorate General of Development Cooperation through the Royal Belgian Institute of Natural Sciences. Laura Kuijpers is supported by the Flemish Ministry of Sciences (EWI, SOFI project IDIS). The funders had no role in study design, data collection and analysis, decision to publish, or preparation of the manuscript, therefore we report no competing interests.

\section{Availability of data and materials}

The dataset(s) supporting the conclusions of this article is (are) included within the article (and its additional file(s).

\section{Authors' contributions}

$D F, H D, J J, D N$ and CVG conceived and designed the study. DF, LMFK, MFP, $\mathrm{HDB}, \mathrm{OL}, \mathrm{SB}$ and RV collected the data. LMFK, DF, JJ, SB, WM and PJC analyzed the data. EVe, CVG, DN, Eva and HD contributed reagents, materials and analysis tools. LMFK, DF and JJ contributed to the writing of the manuscript. LMFK, DF, MFP, HDB, OL, EVa, SB, WM, PJC, RV, HD, CVG, EVe, DN and $J$ J critically reviewed the manuscript and made suggestions of improvements. All authors read and approved the final manuscript.

\section{Competing interests}

The authors declare that they have no competing interests.

\section{Ethics approval and consent to participate}

Ethical approval for the Microbiological Surveillance Study was granted by the Institutional Review Board of the ITM, the Ethics Committee of Antwerp University, and the Ministry of Health of the DRC. In view of the absence of an Ethical committee in Kisangani at the time of the study, written authorization to retrospectively review the patient files was obtained from the Medical Inspector of the Oriental Province (Reference number: $N^{0} 701 /$ DPS/PERS/872/2012) who represents the highest health authority as well as from the Chief Medical Officer of the Haut-Uélé district (Reference number: 771/23/ISP/HU.O/2012).

\section{Author details}

${ }^{1}$ Department of Pediatrics, University Hospital of Kisangani, Kisangani, the Democratic Republic of the Congo. ${ }^{2}$ Department of Clinical Sciences, Institute of Tropical Medicine, Antwerp, Belgium. ${ }^{3}$ Department of Microbiology and Immunology, KU Leuven, Leuven, Belgium. ${ }^{4}$ Department of Clinical Microbiology, National Institute for Biomedical Research, Kinshasa, the Democratic Republic of the Congo. ${ }^{5}$ General Referral Hospital of Kabondo, Kisangani, the Democratic Republic of the Congo. ${ }^{6}$ Belgian National Centre for Salmonella, Scientific Institute of Public Health, Brussels, Belgium. ${ }^{7}$ Department of Pediatrics, University Hospital of Leuven, KU Leuven, Leuven, Belgium. ${ }^{8}$ OD Taxonomy \& Phylogeny, Royal Belgian Institute of Natural Sciences, Brussels, Belgium. ${ }^{9}$ Evolutionary Ecology, University of Antwerp, Antwerp, Belgium.

Received: 11 November 2015 Accepted: 1 June 2016

Published online: 10 June 2016

References

1. Gordon MA, Graham SM, Walsh AL, Wilson L, Phiri A, Molyneux E, et al. Epidemics of invasive Salmonella enterica serovar enteritidis and S-enterica serovar typhimurium infection associated with multidrug resistance among adults and children in Malawi. Clin Infect Dis. 2008;46(7):963-9.

2. Feasey NA, Dougan G, Kingsley RA, Heyderman RS, Gordon MA. Invasive non-typhoidal salmonella disease: an emerging and neglected tropical disease in Africa. Lancet. 2012;379(9835):2489-99.

3. Graham SM, English M. Non-typhoidal salmonellae: a management challenge for children with community-acquired invasive disease in tropical African countries. Lancet. 2009;373(9659):267-9.

4. Morpeth SC, Ramadhani HO, Crump JA. Invasive non-typhi salmonella disease in Africa. Clin Infect Dis. 2009;49(4):606-11. 
5. MacLennan CA. Out of Africa. Links between invasive nontyphoidal salmonella disease, typhoid fever, and malaria. Clin Infect Dis. 2014;58(5):648-50.

6. Lunguya O, Lejon V, Phoba MF, Bertrand S, Vanhoof R, Glupczynski Y, et al. Antimicrobial resistance in invasive Non-typhoid salmonella from the Democratic Republic of the Congo: emergence of decreased fluoroquinolone susceptibility and extended-spectrum beta lactamases. PLoS Negl Trop Dis. 2013;7(3):e2103.

7. Biggs HM, Lester R, Nadjm B, Mtove G, Todd JE, Kinabo GD, et al. Invasive salmonella infections in areas of high and Low malaria transmission intensity in Tanzania. Clin Infect Dis. 2014;58(5):638-47.

8. Kleinschmidt I, Schwabe C, Benavente L, Torrez M, Ridl FC, Segura JL, et al. Marked increase in child survival after four years of intensive malaria control. Am J Trop Med Hyg. 2009;80(6):882-8.

9. Mabey DCW, Brown A, Greenwood BM. Plasmodium-falciparum malaria and salmonella infections in gambian children. J Infect Dis. 1987;155(6):1319-21.

10. Gordon MA, Banda HT, Gondwe M, Gordon SB, Boeree MJ, Walsh AL, et al. Non-typhoidal salmonella bacteraemia among HIV-infected Malawian adults: high mortality and frequent recrudescence. AIDS. 2002;16(12):1633-41.

11. Mtove G, Amos B, von Seidlein L, Hendriksen I, Mwambuli A, Kimera J, et al. Invasive salmonellosis among children admitted to a rural tanzanian hospital and a comparison with previous studies. PLoS One. 2010;5(2):e9244.

12. Kariuki S, Revathi G, Kariuki N, Kiiru J, Mwituria J, Hart CA. Characterisation of community acquired non-typhoidal Salmonella from bacteraemia and diarrhoeal infections in children admitted to hospital in Nairobi, Kenya. BMC Microbiol. 2006;6:101.

13. Dione MM, Ikumapayi UN, Saha D, Mohammed NI, Geerts S, leven M, et al. Clonal differences between non-typhoidal salmonella (NTS) recovered from children and animals living in close contact in the Gambia. PLoS Negl Trop Dis. 2011;5:5.

14. Mandomando I, Macete E, Sigauque B, Morais L, Quinto L, Sacarlal J, et al. Invasive non-typhoidal Salmonella in Mozambican children. Trop Med Int Health. 2009;14(12):1467-74.

15. Phoba MF, Lunguya O, Mayimon DV, di Mputu PL, Bertrand S, Vanhoof R, Verhaegen J, Van Geet C, Muyembe JJ, Jacobs J. Multidrug-resistant salmonella enterica, Democratic Republic of the Congo. Emerg Infect Dis. 2012;18(10):1692-4.

16. Phoba MF, De Boeck H, Ifeka BB, Dawili J, Lunguya O, Vanhoof $R$, et al, Epidemic increase in Salmonella bloodstream infection in children, Bwamanda, the Democratic Republic of Congo. Eur J Clin Microbiol Infect Dis. 2014;33(1):79-87.

17. MSF. MSF launches massive malaria response across three provinces. 2012. [http://www.doctorswithoutborders.org/news-stories/field-news/ drc-msf-launches-massive-malaria-response-across-three-provinces] Accessed 04/03/2016.

18. Gouvernement de la Province Orientale. Plan quinquennal de croissance et de l'emploi 2011-2015. 2011.

19. World Health Organization (WHO). World malaria report 2013. 2013.

20. UNICEF. UNICEF humanitarian action update: Democratic Republic of the Congo. 2012.

21. United Nations Office for the Coordination of Humanitarian Affairs (OCHA) Humanitarian action in the Democratic Republic of the Congo (DRC) weekly bulletin 10 February 2012. 2012

22. Lunguya O, Phoba MF, Mundeke SA, Bonebe E, Mukadi P, Muyembe JJ, et al. The diagnosis of typhoid fever in the Democratic Republic of the Congo. Trans R Soc Trop Med Hyg. 2012;106(6):348-55.

23. Kalonji LM, Post A, Phoba MF, Falay D, Ngbonda D, Muyembe JJ, et al. Invasive Salmonella Infections at Multiple Surveillance Sites in the Democratic Republic of the Congo, 2011-2014. Clin Infect Dis. 2015;61(Supplement 4):S346-53.

24. Grimont PA, Weill FX. Antigenic formulae of the Salmonella serovars. 9th ed. 2007.

25. World Health Organization (WHO). Background document: the diagnosis, treatment and prevention of typhoid fever. 2003.

26. Griggs DJ, Gensberg K, Piddock LJV. Mutations in gyrA gene of quinoloneresistant Salmonella serotypes isolated from humans and animals. Antimicrob Agents Chemother. 1996;40(4):1009-13.

27. Cavaco LM, Aarestrup FM. Evaluation of Quinolones for Use in Detection of Determinants of Acquired Quinolone Resistance, Including the New Transmissible Resistance Mechanisms qnrA, qnrB, qnrS, and aac( $\left.6^{\prime}\right) \mathrm{lb}-\mathrm{cr}$, in Escherichia coli and Salmonella enterica and Determinations of Wild-Type Distributions. J Clin Microbiol. 2009;47(9):2751-8.

28. Hopkins KL, Peters TM, de Pinna E, Wain J. Standardisation of multilocus variable-number tandem-repeat analysis (MLVA) for subtyping of Salmonella enterica serovar Enteritidis. Eurosurveillance. 2011;16(32):2-12.

29. Bertrand S, De Bex GDL, Wildemauwe C, Lunguya O, Phoba MF, Ley B, et al. Multi Locus Variable-Number Tandem Repeat (MLVA) Typing Tools Improved the Surveillance of Salmonella Enteritidis: A 6 Years Retrospective Study. PLoS One. 2015;10:2.

30. Danoff-Burg J. Measuring Biological Diversity. 2003. http://www.columbia. edu/itc/cerc/danoff-burg/MBD_Links.html Accessed 21/10/2015.

31. World Health Organization (WHO). Manual of Basic Techniques for a Health Laboratory. 1980.

32. Ministère de la Santé RDC. Bonnes pratiques transfusionnelles. 2005.

33. World Health Organization (WHO). Pocket book of Hospital care for children. Guidelines for the management of common childhood illnesses. 2013.

34. Global Information and Early Warning System. Food and Agriculture Organization of the United Nations. Interpolated Estimated Dekadal Rainfall. Democratic Republic of the Congo. 2015.

35. Bronzan RN, Taylor TE, Mwenechanya J, Tembo M, Kayira K, Bwanaisa L, et al. Bacteremia in Malawian children with severe malaria: prevalence, etiology, HIV coinfection, and outcome. J Infect Dis. 2007;195(6):895-904.

36. Sigauque B, Roca A, Mandomando I, Morais L, Quinto L, Sacarlal J, et al. Community-acquired bacteremia among children admitted to a rural hospital in Mozambique. Pediatr Infect Dis J. 2009;28(2):108-13.

37. Enwere G, Biney E, Cheung Y, Zaman SMA, Okoko B, Oluwalana C, et al. Epidemiologic and clinical characteristics of community-acquired invasive bacterial infections in children aged 2-29 months in the Gambia. Pediatr Infect Dis J. 2006;25(8):700-5.

38. Feasey NA, Archer BN, Heyderman RS, Sooka A, Dennis B, Gordon MA, et al. Typhoid fever and invasive nontyphoid salmonellosis, Malawi and south Africa. Emerg Infect Dis. 2010;16(9):1448-51.

39. Brent AJ, Oundo JO, Mwangi I, Ochola L, Lowe B, Berkley JA. Salmonella bacteremia in Kenyan children. Pediatr Infect Dis J. 2006;25(3):230-6.

40. Church J, Maitland K. Invasive bacterial co-infection in African children with Plasmodium falciparum malaria: a systematic review. BMC Med. 2014;12:31.

41. Takem EN, Roca A, Cunnington A. The association between malaria and non-typhoid Salmonella bacteraemia in children in sub-Saharan Africa: a literature review. Malar J. 2014;13:400.

42. Walsh AL, Molyneux EM, Kabudula M, Phiri AJ, Molyneux ME, Graham SM. Bacteraemia following blood transfusion in Malawian children: predominance of Salmonella. Trans R Soc Trop Med Hyg. 2002;96(3):276-7.

43. Cunnington AJ, de Souza JB, Walther M, Riley EM. Malaria impairs resistance to Salmonella through heme- and heme oxygenase-dependent dysfunctional granulocyte mobilization. Nat Med. 2012;18(1):120-7.

44. Roux CM, Butler BP, Chau JY, Paixao TA, Cheung KW, Santos RL, et al. Both hemolytic anemia and malaria parasite-specific factors increase susceptibility to nontyphoidal salmonella enterica serovar typhimurium infection in mice. Infect Immun. 2010;78(4):1520-7.

45. Cunnington AJ, Njie M, Correa S, Takem EN, Riley EM, Walther M. Prolonged neutrophil dysfunction after plasmodium falciparum malaria is related to hemolysis and heme oxygenase-1 induction. J Immunol. 2012;189(11):5336-46.

46. Malaria Atlas Project. The spatial distribution of Plasmodium falciparum malaria stratified by endemicity class map in 2010 in Democratic Republic of the Congo. 2010. http://www.map.ox.ac.uk/browse-resources/endemicity/ Pf_class/COD/ Accessed 07/03/2016.

47. World Health Organization (WHO). Sickle-cell disease: A strategy for the WHO African region. 2010.

48. Ley B, Le Hello S, Lunguya O, Lejon V, Muyembe JJ, Weill FX, et al. Invasive salmonella enterica serotype typhimurium infections, democratic Republic of the Congo, 2007-2011. Emerg Infect Dis. 2014;20(4):701-4.

49. Kingsley RA, Msefula CL, Thomson NR, Kariuki S, Holt KE, Gordon MA, et al. Epidemic multiple drug resistant Salmonella Typhimurium causing invasive disease in sub-Saharan Africa have a distinct genotype. Genome Res. 2009:19(12):2279-87. 